\title{
Non-Linear Invariance of Black Hole Entropy
}

\author{
Alessio Marrani* \\ Centro Studi e Ricerche "Enrico Fermi", \\ Via Panisperna 89A, I-00184, Roma, IT \\ and Dipartimento di Fisica e Astronomia "Galileo Galilei”, Università di Padova, \\ and INFN - sez. di Padova, Via Marzolo 8, I-35131 Padova, IT \\ E-mail: marraniepd.infn.it
}

Freudenthal duality is an anti-involutive, non-linear map acting on symplectic spaces. It generally holds in four-dimensional Maxwell-Einstein theories coupled to a non-linear sigma model of scalar fields. It is here reviewed, with some emphasis on its relation to the $U$-duality Lie groups of type $E_{7}$ in extended supergravity theories.

The European Physical Society Conference on High Energy Physics

5-12 July

Venice, Italy

${ }^{*}$ Speaker. 


\section{Freudenthal Duality}

We start and consider the following Lagrangian density in four dimensions (cfr. e.g. [1]):

$$
\mathscr{L}=-\frac{R}{2}+\frac{1}{2} g_{i j}(\varphi) \partial_{\mu} \varphi^{i} \partial^{\mu} \varphi^{j}+\frac{1}{4} I_{\Lambda \Sigma}(\varphi) F_{\mu \nu}^{\Lambda} F^{\Sigma \mid \mu \nu}+\frac{1}{8 \sqrt{-G}} R_{\Lambda \Sigma}(\varphi) \varepsilon^{\mu \nu \rho \sigma} F_{\mu \nu}^{\Lambda} F_{\rho \sigma}^{\Sigma},
$$

describing Einstein gravity coupled to Maxwell (Abelian) vector fields and to a non-linear sigma model of scalar fields (with no potential); note that $\mathscr{L}$ may -but does not necessarily need to be conceived as the bosonic sector of $D=4$ (ungauged) supergravity theory. Out of the Abelian two-form field strengths $F^{\Lambda}$ 's, one can define their duals $G_{\Lambda}$, and construct a symplectic vector :

$$
H:=\left(F^{\Lambda}, G_{\Lambda}\right)^{T},{ }^{*} G_{\Lambda \mid \mu v}:=2 \frac{\delta \mathscr{L}}{\delta F^{\Lambda \mid \mu \nu}} .
$$

We then consider the simplest solution of the equations of motion deriving from $\mathscr{L}$, namely a static, spherically symmetric, asymptotically flat, dyonic extremal black hole with metric [2]

$$
d s^{2}=-e^{2 U(\tau)} d t^{2}+e^{-2 U(\tau)}\left[\frac{d \tau^{2}}{\tau^{4}}+\frac{1}{\tau^{2}}\left(d \theta^{2}+\sin \theta d \psi^{2}\right)\right],
$$

where $\tau:=-1 / r$. Thus, the two-form field strengths and their duals can be fluxed on the twosphere at infinity $S_{\infty}^{2}$ in such a background, respectively yielding the electric and magnetic charges of the black hole itself, which can be arranged in a symplectic vector $\mathscr{Q}$ :

$$
\begin{aligned}
p^{\Lambda} & :=\frac{1}{4 \pi} \int_{S_{\infty}^{2}} F^{\Lambda}, \quad q_{\Lambda}:=\frac{1}{4 \pi} \int_{S_{\infty}^{2}} G_{\Lambda}, \\
\mathscr{Q} & :=\left(p^{\Lambda}, q_{\Lambda}\right)^{T} .
\end{aligned}
$$

Then, by exploiting the symmetries of the background (1.3), the Lagrangian (1.1) can be dimensionally reduced from $D=4$ to $D=1$, obtaining a 1-dimensional effective Lagrangian (' $:=$ $d / d \tau)[3]:$

$$
\mathscr{L}_{D=1}=\left(U^{\prime}\right)^{2}+g_{i j}(\varphi) \varphi^{i \prime} \varphi^{j \prime}+e^{2 U} V_{B H}(\varphi, \mathscr{Q})
$$

along with the Hamiltonian constraint [3]

$$
\left(U^{\prime}\right)^{2}+g_{i j}(\varphi) \varphi^{i \prime} \varphi^{j \prime}-e^{2 U} V_{B H}(\varphi, \mathscr{Q})=0 .
$$

The so-called "effective black hole potential" $V_{B H}$ appearing in (1.6) and (1.7) is defined as [3]

$$
V_{B H}(\varphi, \mathscr{Q}):=-\frac{1}{2} \mathscr{Q}^{T} \mathscr{M}(\varphi) \mathscr{Q},
$$

in terms of the symplectic and symmetric matrix [1]

$$
\begin{aligned}
& \mathscr{M}:=\left(\begin{array}{cc}
\mathbb{I} & -R \\
0 & \mathbb{I}
\end{array}\right)\left(\begin{array}{cc}
I & 0 \\
0 & I^{-1}
\end{array}\right)\left(\begin{array}{cc}
\mathbb{I} & 0 \\
-R & \mathbb{I}
\end{array}\right)=\left(\begin{array}{cc}
I+R I^{-1} R & -R I^{-1} \\
-I^{-1} R & I^{-1}
\end{array}\right), \\
& \mathscr{M}^{T}=\mathscr{M} ; \mathscr{M} \Omega \mathscr{M}=\Omega,
\end{aligned}
$$


where $\mathbb{I}$ denotes the identity, and $R(\varphi)$ and $I(\varphi)$ are the scalar-dependent matrices occurring in (1.1); moreover, $\Omega$ stands for the symplectic metric $\left(\Omega^{2}=-\mathbb{I}\right)$. Note that, regardless of the invertibility of $R(\varphi)$ and as a consequence of the physical consistence of the kinetic vector matrix $I(\varphi)$, $\mathscr{M}$ is negative-definite; thus, the effective black hole potential (1.8) is positive-definite.

By virtue of the matrix $\mathscr{M}$, one can introduce a (scalar-dependent) anti-involution $\mathscr{S}$ in any Maxwell-Einstein-scalar theory described by (1.1) with a symplectic structure $\Omega$, as follows :

$$
\begin{aligned}
\mathscr{S}(\varphi) & :=\Omega \mathscr{M}(\varphi) ; \\
\mathscr{S}^{2}(\varphi) & =\Omega \mathscr{M}(\varphi) \Omega \mathscr{M}(\varphi)=\Omega^{2}=-\mathbb{I} ;
\end{aligned}
$$

in turn, this allows to define an anti-involution on the dyonic charge vector $\mathscr{Q}$, which has been called (scalar-dependent) Freudenthal duality [4, 5, 6]:

$$
\begin{aligned}
\mathfrak{F}(\mathscr{Q} ; \varphi) & :=-\mathscr{S}(\varphi) \mathscr{Q} ; \\
\mathfrak{F}^{2} & =-\mathbb{I}, \quad(\forall\{\varphi\}) .
\end{aligned}
$$

By recalling (1.8) and (1.11), the action of $\mathfrak{F}$ on $\mathscr{Q}$, defining the so-called ( $\varphi$-dependent) Freudenthal dual of $\mathscr{Q}$ itself, can be related to the symplectic gradient of the effective black hole potential $V_{B H}$ :

$$
\mathfrak{F}(\mathscr{Q} ; \varphi)=\Omega \frac{\partial V_{B H}(\varphi, \mathscr{Q})}{\partial \mathscr{Q}} .
$$

Through the attractor mechanism [7], all this enjoys an interesting physical interpretation when evaluated at the (unique) event horizon of the extremal black hole (1.3) (denoted below by the subscript " $H$ "); indeed

$$
\begin{aligned}
\partial_{\varphi} V_{B H} & =0 \Leftrightarrow \lim _{\tau \rightarrow-\infty} \varphi^{i}(\tau)=\varphi_{H}^{i}(\mathscr{Q}) ; \\
S_{B H}(\mathscr{Q}) & =\frac{A_{H}}{4}=\left.\pi V_{B H}\right|_{\partial_{\varphi} V_{B H}=0}=-\frac{\pi}{2} \mathscr{Q}^{T} \mathscr{M}_{H}(\mathscr{Q}) \mathscr{Q},
\end{aligned}
$$

where $S_{B H}$ and $A_{H}$ respectively denote the Bekenstein-Hawking entropy [8] and the area of the horizon of the extremal black hole, and the matrix horizon value $\mathscr{M}_{H}$ is defined as

$$
\mathscr{M}_{H}(\mathscr{Q}):=\lim _{\tau \rightarrow-\infty} \mathscr{M}(\varphi(\tau)) .
$$

Correspondingly, one can define the (scalar-independent) horizon Freudenthal duality $\mathfrak{F}_{H}$ as the horizon limit of (1.13) :

$$
\widetilde{\mathscr{Q}} \equiv \mathfrak{F}_{H}(\mathscr{Q}):=\lim _{\tau \rightarrow-\infty} \mathfrak{F}(\mathscr{Q} ; \varphi(\tau))=-\Omega \mathscr{M}_{H}(\mathscr{Q}) \mathscr{Q}=\frac{1}{\pi} \Omega \frac{\partial S_{B H}(\mathscr{Q})}{\partial \mathscr{Q}} .
$$

Remarkably, the (horizon) Freudenthal dual of $\mathscr{Q}$ is nothing but $(1 / \pi$ times) the symplectic gradient of the Bekenstein-Hawking black hole entropy $S_{B H}$; this latter, from dimensional considerations, is only constrained to be an homogeneous function of degree two in $\mathscr{Q}$. As a result, $\widetilde{\mathscr{Q}}=\widetilde{\mathscr{Q}}(\mathscr{Q})$ is generally a complicated (non-linear) function, homogeneous of degree one in $\mathscr{Q}$.

It can be proved that the entropy $S_{B H}$ itself is invariant along the flow in the charge space $\mathscr{Q}$ defined by the symplectic gradient (or, equivalently, by the horizon Freudenthal dual) of $\mathscr{Q}$ itself :

$$
S_{B H}(\mathscr{Q})=S_{B H}\left(\mathfrak{F}_{H}(\mathscr{Q})\right)=S_{B H}\left(\frac{1}{\pi} \Omega \frac{\partial S_{B H}(\mathscr{Q})}{\partial \mathscr{Q}}\right)=S_{B H}(\widetilde{\mathscr{Q}})
$$


It is here worth pointing out that this invariance is pretty remarkable : the (semi-classical) Bekenstein-Hawking entropy of an extremal black hole turns out to be invariant under a generally non-linear map acting on the black hole charges themselves, and corresponding to a symplectic gradient flow in their corresponding vector space.

For other applications and instances of Freudenthal duality, see $[9,10,11,12]$.

\section{Groups of Type $E_{7}$}

The concept of Lie groups of type $E_{7}$ as introduced in the 60s by Brown [13], and then later developed e.g. by $[14,15,16,17,18]$. Starting from a pair $(G, \mathbf{R})$ made of a Lie group $G$ and its faithful representation $\mathbf{R}$, the three axioms defining $(G, \mathbf{R})$ as a group of type $E_{7}$ read as follows :

1. Existence of a (unique) symplectic invariant structure $\Omega$ in $\mathbf{R}$ :

$$
\exists ! \Omega \equiv \mathbf{1} \in \mathbf{R} \times{ }_{a} \mathbf{R},
$$

which then allows to define a symplectic product $\langle\cdot, \cdot\rangle$ among two vectors in the representation space $\mathbf{R}$ itself :

$$
\left\langle Q_{1}, Q_{2}\right\rangle:=Q_{1}^{M} Q_{2}^{N} \Omega_{M N}=-\left\langle Q_{2}, Q_{1}\right\rangle .
$$

2. Existence of (unique) rank-4 completely symmetric invariant tensor ( $K$-tensor) in $\mathbf{R}$ :

$$
\exists ! K \equiv \mathbf{1} \in(\mathbf{R} \times \mathbf{R} \times \mathbf{R} \times \mathbf{R})_{s},
$$

which then allows to define a degree-4 invariant polynomial $I_{4}$ in $\mathbf{R}$ itself :

$$
I_{4}:=K_{M N P Q} Q^{M} Q^{N} Q^{P} Q^{Q} .
$$

3. Defining a triple map $T$ in $\mathbf{R}$ as

$$
\begin{aligned}
T & : \mathbf{R} \times \mathbf{R} \times \mathbf{R} \rightarrow \mathbf{R} \\
\left\langle T\left(Q_{1}, Q_{2}, Q_{3}\right), Q_{4}\right\rangle & :=K_{M N P Q} Q_{1}^{M} Q_{2}^{N} Q_{3}^{P} Q_{4}^{Q},
\end{aligned}
$$

it holds that

$$
\left\langle T\left(Q_{1}, Q_{1}, Q_{2}\right), T\left(Q_{2}, Q_{2}, Q_{2}\right)\right\rangle=\left\langle Q_{1}, Q_{2}\right\rangle K_{M N P Q} Q_{1}^{M} Q_{2}^{N} Q_{2}^{P} Q_{2}^{Q}
$$

This property makes a group of type $E_{7}$ amenable to a description as an automorphism group of a Freudenthal triple system (or, equivalently, as the conformal groups of the underlying Jordan triple system - whose a Jordan algebra is a particular case - ).

All electric-magnetic duality $\left(U\right.$-duality $\left.{ }^{1}\right)$ groups of $\mathscr{N} \geqslant 2$-extended $D=4$ supergravity theories with symmetric scalar manifolds are of type $E_{7}$. Among these, degenerate groups of type $E_{7}$ are those in which the $K$-tensor is actually reducible, and thus $I_{4}$ is the square of a quadratic

\footnotetext{
${ }^{1}$ Here $U$-duality is referred to as the "continuous" symmetries of [19]. Their discrete versions are the $U$-duality non-perturbative string theory symmetries introduced by Hull and Townsend [20].
} 
invariant polynomial $I_{2}$. In fact, in general, in theories with electric-magnetic duality groups of type $E_{7}$ holds that

$$
S_{B H}=\pi \sqrt{\left|I_{4}(\mathscr{Q})\right|}=\pi \sqrt{\left|K_{M N P Q} \mathscr{Q}^{M} \mathscr{Q}^{N} \mathscr{Q}^{P} \mathscr{Q}^{Q}\right|},
$$

whereas in the case of degenerate groups of type $E_{7}$ it holds that $I_{4}(\mathscr{Q})=\left(I_{2}(\mathscr{Q})\right)^{2}$, and therefore the latter formula simplifies to

$$
S_{B H}=\pi \sqrt{\left|I_{4}(\mathscr{Q})\right|}=\pi\left|I_{2}(\mathscr{Q})\right| .
$$

Simple, non-degenerate groups of type $E_{7}$ relevant to $\mathscr{N} \geqslant 2$-extended $D=4$ supergravity theories with symmetric scalar manifolds are listed e.g. in Table 1 of [21].

Semi-simple, non-degenerate groups of type $E_{7}$ of the same kind are given by $G=S L(2, \mathbb{R}) \times$ $S O(2, n)$ and $G=S L(2, \mathbb{R}) \times S O(6, n)$, with $\mathbf{R}=(\mathbf{2}, \mathbf{2}+\mathbf{n})$ and $\mathbf{R}=(\mathbf{2}, \mathbf{6}+\mathbf{n})$, respectively relevant for $\mathscr{N}=2$ and $\mathscr{N}=4$ supergravity.

Moreover, degenerate (simple) groups of type $E_{7}$ relevant to the same class of theories are $G=U(1, n)$ and $G=U(3, n)$, with complex fundamental representations $\mathbf{R}=\mathbf{n}+\mathbf{1}$ and $\mathbf{R}=\mathbf{3}+\mathbf{n}$, respectively relevant for $\mathscr{N}=2$ and $\mathscr{N}=3$ supergravity [17].

The classification of groups of type $E_{7}$ is still an open problem, even if some progress have been recently made $e . g$. in [22] (in particular, $c f r$. Table D therein).

\section{References}

[1] P. Breitenlohner, G. W. Gibbons, and D. Maison, Four-Dimensional Black Holes from Kaluza-Klein Theories, Commun. Math. Phys. 120, 295 (1988).

[2] A. Papapetrou, A static solution of the equations of the gravitational field for an arbitrary charge distribution, Proc. R. Irish Acad. A51, 191 (1947). S. D. Majumdar, A Class of Exact Solutions of Einstein's Field Equations, Phys. Rev. 72, 930 (1947).

[3] S. Ferrara, G. W. Gibbons, and R. Kallosh, Black holes and critical points in moduli space, Nucl. Phys. B500, 75 (1997), hep-th/9702103.

[4] L. Borsten, D. Dahanayake, M. J. Duff, and W. Rubens, Black holes admitting a Freudenthal dual, Phys. Rev. D80 (2009) 026003, arXiv: 0903.5517 [hep-th] .

[5] S. Ferrara, A. Marrani, and A. Yeranyan, Freudenthal Duality and Generalized Special Geometry, Phys. Lett. B701 (2011) 640, arXiv:1102.4857 [hep-th].

[6] L. Borsten, M. J. Duff, S. Ferrara, and A. Marrani, Freudenthal Dual Lagrangians, Class. Quant.Grav. 30 (2013) 235003, arXiv:1212.3254 [hep-th] .

[7] S. Ferrara, R. Kallosh, and A. Strominger, $\mathscr{N}=2$ Extremal Black Holes, Phys. Rev. D52, 5412 (1995), hep-th/9508072. A. Strominger, Macroscopic Entropy of $\mathscr{N}=2$ Extremal Black Holes, Phys. Lett. B383, 39 (1996), hep-th/9602111. S. Ferrara and R. Kallosh, Supersymmetry and Attractors, Phys. Rev. D54, 1514 (1996), hep-th/9602136. S. Ferrara and R. Kallosh, Universality of Supersymmetric Attractors, Phys. Rev. D54, 1525 (1996), hep-th/ 9603090.

[8] S. W. Hawking: Gravitational Radiation from Colliding Black Holes, Phys. Rev. Lett. 26, 1344 (1971). J. D. Bekenstein: Black Holes and Entropy, Phys. Rev. D7, 2333 (1973).

[9] P. Galli, P. Meessen, and T. Ortín, The Freudenthal gauge symmetry of the black holes of $\mathscr{N}=2, d=4$ supergravity, JHEP 1305 (2013) 011, arXiv: 1211.7296 [hep-th] . 
[10] J.J. Fernandez-Melgarejo and E. Torrente-Lujan, $\mathscr{N}=2$ Sugra BPS Multi-center solutions, quadratic prepotentials and Freudenthal transformations, JHEP 1405 (2014) 081, arXiv: 1310.4182 [hep-th].

[11] A. Marrani, C.-X. Qiu, S.-Y. D. Shih, A. Tagliaferro, and B. Zumino, Freudenthal Gauge Theory, JHEP 1303 (2013) 132, arXiv: 1208.0013 [hep-th] .

[12] A. Marrani, P. K. Tripathy, T. Mandal, Supersymmetric Black Holes and Freudenthal Duality, Int.J.Mod.Phys. A32 (2017) no.19n20, 1750114, arXiv:1703.08669 [hep-th] .

[13] R. B. Brown, Groups of Type E 7 , J. Reine Angew. Math. 236, 79 (1969).

[14] K. Meyberg, Eine Theorie der Freudenthalschen Triplesysteme. I, II, Nederl. Akad. Wetensch. Proc. Ser. A71, 162 (1968).

[15] R. S. Garibaldi, Groups of type $E_{7}$ over Arbitrary Fields, Commun. in Algebra 29, 2689 (2001), math/9811056 [math.AG].

[16] S. Krutelevich, Jordan algebras, exceptional groups, and higher composition laws, arXiv:math/ 0411104 .S. Krutelevich, Jordan algebras, exceptional groups, and Bhargava composition, J. of Algebra 314, 924 (2007).

[17] S. Ferrara, R. Kallosh, and A. Marrani, Degeneration of Groups of Type $E_{7}$ and Minimal Coupling in Supergravity, JHEP 1206 (2012) 074, arXiv: 1202.1290 [hep-th] .

[18] A. Marrani, E. Orazi, and F. Riccioni, Exceptional Reductions, J. Phys. A44, 155207 (2011), arXiv:1012.5797 [hep-th].

[19] E. Cremmer and B. Julia, The $\mathscr{N}=8$ Supergravity Theory. 1. The Lagrangian, Phys. Lett. B80, 48 (1978). E. Cremmer and B. Julia, The SO(8) Supergravity, Nucl. Phys. B159, 141 (1979).

[20] C. Hull and P. K. Townsend, Unity of Superstring Dualities, Nucl. Phys. B438, 109 (1995), hep-th/9410167.

[21] A. Marrani, Freudenthal Duality in Gravity: from Groups of Type $E_{7}$ to Pre-Homogeneous Spaces, $\mathrm{p}$ Adic Ultra.Anal.Appl. 7 (2015) 322-331, arXiv:1509.01031 [hep-th] .

[22] S. Garibaldi and R. Guralnick, Simple groups stabilizing polynomials, Forum of Mathematics, Pi (2015), vol. 3, e3, arXiv:1309.6611 [math.GR]. 